\title{
Nutzenpotentiale cyber-physischer Systeme für industrielle Dienstleistungen 4.0
}

\author{
Matthias Herterich · Falk Uebernickel • Walter Brenner
}

Eingegangen: 1. Mai 2015 / Angenommen: 2. Juli 2015 / Online publiziert: 24. Juli 2015

(C) Springer Fachmedien Wiesbaden 2015

Zusammenfassung Das Dienstleistungsgeschäft gewinnt in der produzierenden Industrie, sowie im Maschinen- und Anlagenbau gegenüber dem ausschließlichen Verkauf von industriellen Produkten zunehmend an Bedeutung. Durch die langen Lebenszyklen industrieller Maschinen und Anlagen generieren Produkthersteller und Serviceorganisationen vor allem mit der Sicherstellung des Betriebs und der Durchführung von Instandhaltungen als produktergänzende Dienstleistungen kontinuierliche Einnahmen. Die fortschreitende Digitalisierung und der geschickte Einsatz von cyber-physischen Systemen transformieren dieses Geschäft. Einerseits ergeben sich neue Möglichkeiten zur Steigerung der Effizienz bereits bestehender Prozesse. Andererseits eröffnen sich durch die Verfügbarkeit von Sensordaten und Steuerungsmöglichkeiten der Maschinen und Anlagen aus der Ferne bisher ungeahnte Möglichkeiten zur Umsetzung innovativer industrieller Dienstleistungen. Auf Basis empirischer Daten von 45 konkreten Anwendungsfällen identifiziert dieser Beitrag Nutzenpotentiale cyber-physischer Systeme im Kontext industrieller Dienstleistungen.

Schlüsselwörter Industrielle Dienstleistungen $\cdot$ DienstleistungsGeschäftsmodelle · Cyber-Physische Systeme (CPS) · Anwendungsszenarien · Nutzenpotentiale $\cdot$ Fallstudie

\begin{abstract}
Both managers and researchers agree: Manufacturing as one of the most traditional and conservative industries is undergoing the most fundamental change in decades. Facing shrinking margins in their core business, manufacturing firms of industrial equipment and capital goods started to expand their business by also offering services that complement the traditional product business to address the
\end{abstract}

M. Herterich $(\bowtie) \cdot$ F. Uebernickel $\cdot$ W. Brenner

Institute of Information Management, University of St. Gallen (HSG),

St. Gallen, Schweiz

E-Mail: matthias.herterich@unisg.ch 
long lifecycles of industrial equipment. Digital innovation and industrial equipment with cyber-physical capabilities unlock new opportunities for the industrial service business and pivot the manufacturing industry. With the ability to (1) improve efficiency and quality of existing services, (2) establish new service offerings and (3) transform the service ecosystem, myriad opportunities for service innovation emerge. Based on 45 concrete smart service scenarios, we identify areas of benefit of cyber-physical equipment for the industrial service business.

Keywords Industrial service $\cdot$ Service business models $\cdot$ Cyber-Physical Systems (CPS) - Smart service scenarios · Affordances · Case study

\section{Die Digitalisierung des industriellen Servicegeschäfts ${ }^{1}$}

In den letzten Jahrzehnten hat sich in Europa die wirtschaftliche Struktur stark verändert. Im europäischen Durchschnitt entfällt heute mehr als $70 \%$ der gesamten Wirtschaftsleistung auf den Dienstleistungsbereich (Leimeister 2012; Maglio und Spohrer 2008). Physische Produkte werden zunehmend als Endpunkte und Plattformen betrachtet, um Kunden zusätzliche Dienstleistungen anzubieten (Xu und Ilic 2014). Vor allem im traditionell produktorientierten Maschinen- und Anlagenbau hat das Dienstleistungsgeschäft stark an Bedeutung gewonnen (Oliva und Kallenberg 2003; Ulaga und Reinartz 2011). Unterschiedliche Interessensgruppen wie beispielsweise die Betreiber der Anlagen, die Maschinen- und Anlagenbauer selbst oder auch Organisationen, die für den Technischen Kundendienst (TKD) verantwortlich sind, suchen nach neuen Möglichkeiten zur Reduzierung der teuren Ausfallzeiten industrieller Maschinen. Daneben wollen die Organisationen neue produktbegleitende Dienstleistungen anbieten, um zusätzliches Geschäft zu generieren und sich vom Wettbewerb durch innovative Services abzuheben. Dennoch ist der klassische TKD unabdingbar. Somit ist es weiterhin Ziel, hier die Dienstleistungsproduktivität und -qualität weiter zu erhöhen (Fellmann et al. 2014; Thomas et al. 2014), da die Erbringung von produktnahen Dienstleistungen im Vergleich zum Produktgeschäft stabilere Umsätze verspricht (Ebeling et al. 2014; Gebauer 2008). Darüber hinaus differenziert sich das Dienstleistungsgeschäft gegenüber dem Produktgeschäft durch höhere Margen und einer geringeren Abhängigkeit von Investitionszyklen. Insgesamt ist daher festzustellen, dass sich durch den Trend "Servitization in Manufacturing“ (Ebeling et al. 2014; Lightfoot et al. 2013; Oliva und Kallenberg 2003; Ulaga und Reinartz 2011) Unternehmen im Maschinen- und Anlagenbau zusehends vom Hersteller von industriellen Produkten und Anlagen zum Anbieter hybrider Angebote wandeln. Durch die Kombination von industriellen Gütern einerseits und komplementären, produktbegleitenden Dienstleistungen andererseits ist in diesem Zusammenhang die Rede von ,hybrider Wertschöpfung' (Ulaga und Reinartz 2011).

Die Digitalisierung lässt bisher ungeahnte Nutzenpotentiale zu. Die physische und virtuelle Welt verschmelzen miteinander, indem Sensorik und Aktuatorik in

\footnotetext{
${ }^{1}$ Teile dieses Beitrags basieren auf dem Konferenzbeitrag Herterich, M., Uebernickel, F., and Brenner, W. The Impact of Cyber-Physical Systems on Industrial Services in Manufacturing. Procedia CIRP, (2015).
} 
die Maschinen und Anlagen integriert werden. Da der Maschinen- und Anlagenbau durch große Investitionsvolumina gekennzeichnet ist, fällt die Ergänzung industrieller Maschinen und Anlagen durch cyber-physische Komponenten monetär nicht so sehr ins Gewicht, wie dies beispielsweise im Endkundengeschäft mit Haushaltselektronik und ,weißer Ware“ der Fall ist. In Kombination mit entsprechenden BackendSystemen zur Auswertung der anfallenden Datenmengen, sowie die Integration der Möglichkeit der Steuerung in bestehende Geschäftsprozesse, entstehen sogenannte Cyber-physische Systeme (CPS). Obwohl die Forschung, die sich mit CPS beschäftigt, eine technische Perspektive einnimmt, entscheiden im industriellen Bereich oft Kosten-Nutzen Analysen über den produktiven Einsatz (Schoch und Strassner 2003). Durch digitale Produkt-Innovation (Fichman et al. 2014; Yoo et al. 2010) ergeben sich gerade für die Entwicklung innovativer industrieller Dienstleistungen vorher ungeahnte Nutzenpotentiale (Barrett et al. 2015; Lusch und Nambisan 2015), sowie neue Service-Geschäftsmodelle (Reinheimer und Strahringer 2014). Um die Nutzenpotentiale cyber-physischer Maschinen und Anlagen im Kontext des industriellen Servicegeschäfts genauer zu verstehen und gegeneinander abzugrenzen, wurden elf repräsentative Fallstudien mit Maschinen- und Anlagenbauern, Service-Gesellschaften und Betreibern von industriellem Equipment durchgeführt. Auf Basis 45 konkret im Maschinen- und Anlagenbau identifizierter Dienstleistungsszenarien wird ein Ordnungsrahmen vorgestellt, der Nutzenpotentiale für den Einsatz von CPS vorstellt. Für die abstrahierten Nutzenpotentiale werden beispielhaft konkrete Dienstleistungsszenarien aufgezeigt, die durch digitale Produktinnovation im Sinne cyber-physischer Systeme ermöglicht werden. Durch die Fokussierung des vorliegenden Beitrags auf das industrielle Dienstleistungsgeschäft sind die direkten operativen Potentiale, welche für die Betreiber industrieller Maschinen und Anlagen entstehen, nicht Gegenstand der Betrachtungen.

Die Struktur dieses Beitrags gliedert sich in sechs Abschnitte. Nach der einleitenden Motivation für das Thema wird im zweiten Abschnitt ein grundlegendes Verständnis für zentrale Begriffe und Konzepte geschaffen. Der dritte Abschnitt zeigt die verwendete Methodik auf. Im vierten Abschnitt werden die identifizierten CPS Nutzenpotentiale für das industrielle Servicegeschäft vorgestellt. Hierbei wird eine Dienstleistungsperspektive eingenommen. Nutzenpotentiale aus Sicht der Anlagenbetreiber werden weitestgehend ausgeklammert. Die Ergebnisse werden anschließend diskutiert. Dabei werden Implikationen und konkrete Handlungsempfehlungen für die Unternehmenspraxis aufgezeigt. Der Beitrag schließt mit der Diskussion weiterführender Fragestellungen, die sich aus den Ergebnissen dieses Beitrags ergeben.

\section{Industrielle Dienstleistungen für digitalisierte Maschinen und Anlagen}

\subsection{Charakteristika industrieller Maschinen und Anlagen}

Industrielle Güter zeichnen sich durch hohe Anschaffungskosten und eine verhältnismäßig lange Lebensdauer aus. Den Lebenszyklus industrieller Maschinen und Anlagen kann man in drei Phasen unterteilen (Blinn et al. 2008; Kiritsis 2011). Tabelle 1 gibt einen Überblick über die Lebensphasen industrieller Güter. Es wird schnell deut- 
Tab. 1 Lebenszyklusphasen industrieller Güter (Kiritsis 2011)

\begin{tabular}{|c|c|c|}
\hline Produktlebensphase & Beschreibung & Involvierte Akteure \\
\hline $\begin{array}{l}\text { Beginning of life (BOL) } \\
\text { Anfang der Produktlebensphase/ } \\
\text { Produktentwicklung }\end{array}$ & $\begin{array}{l}\text { Konzeptualisierung, Definition und } \\
\text { Realisation der industriellen Güter }\end{array}$ & $\begin{array}{l}\text { Maschinen und Anlagen- } \\
\text { bauer, Lieferant und } \\
\text { Innovationspartner }\end{array}$ \\
\hline $\begin{array}{l}\text { Middle of life (MOL) } \\
\text { Mitte der Produktlebensphase }\end{array}$ & $\begin{array}{l}\text { Nutzung, Service und Wartung der in- } \\
\text { dustriellen Güter beim Betreiber vor Ort }\end{array}$ & $\begin{array}{l}\text { Betreiber von industriellen } \\
\text { Maschinen und Anlagen } \\
\text { und Servicegesellschaften }\end{array}$ \\
\hline $\begin{array}{l}\text { End of life (EOL) } \\
\text { Ende der Produktlebensphase/ } \\
\text { Produktentsorgung }\end{array}$ & $\begin{array}{l}\text { Wiederverwendung von Komponenten } \\
\text { oder gesamten industriellen Maschinen } \\
\text { oder Anlagen, Sanierung, Entsorgung } \\
\text { mit oder ohne Verbrennung }\end{array}$ & $\begin{array}{l}\text { Maschinen- und } \\
\text { Anlagenbauer }\end{array}$ \\
\hline
\end{tabular}

lich, dass die Lebenszyklusphase, in der die Maschinen oder Anlagen genutzt werden, mit Abstand den längsten Zeitraum darstellt.

\subsection{Industrielle Dienstleistungen und Dienstleistungsorientierung im Maschinen- und Anlagenbau}

Für Industrieunternehmen im traditionell produktorientierten Maschinen- und Anlagenbau hat das Dienstleistungsgeschäft in den letzten Jahren eine zunehmend wichtigere Rolle eingenommen (Ebeling et al. 2014; Oliva und Kallenberg 2003; Ulaga und Reinartz 2011). Die Ursache hierfür ist im konstanteren Umsatzpotential von industriellen Dienstleistungen, sowie einer Marktsättigung für teure industrielle Investitionsgüter zu sehen. Industrielle Dienstleistungen werden oft ergänzend zu physischen Produkten angeboten. Beispielsweise wirkt sich möglicher Ausfall direkt negativ auf die Wertschöpfungsprozesse der Betreiber aus. Deshalb ist es den Betreibern wichtig, dass ein reibungsloser Betrieb sichergestellt wird. Da der Anschaffungspreis in der Regel nur einen Bruchteil der Gesamtkosten über den gesamten Lebenszyklus hinweg ausmacht, fokussieren sich Maschinen- und Anlagenbauer verstärkt auf das Geschäft mit Reparatur- und Wartungsdienstleistungen. In diesem Zusammenhang ist von hybriden Dienstleistungen oder Produkt-Service Systemen die Rede. Eine hybride Dienstleistung bezeichnet eine Kombination von gemeinsam angebotenen Dienstleistungen und Sachgütern.

Wegen der Heterogenität gibt es keine einheitliche wissenschaftliche Definition des Begriffs „Dienstleistung“. Deshalb konstituieren die Immaterialität, Nichtlagerfähigkeit, Simultanität von Produktion und Konsum, sowie die Integration externer Faktoren (bspw. Menschen oder Objekte) in den Prozess der Leistungserbringung den Dienstleistungsbegriff (Böhmann et al. 2014; Vargo und Lusch 2008).

Die Digitalisierung trägt dazu bei, dass industrielle Maschinen und Anlagen zunehmend mit digitalen Komponenten und Konnektivität ausgestattet werden. Dadurch ergeben sich neue Möglichkeiten, innovative Dienstleistungen anzubieten oder die Dienstleistungsproduktivität und -qualität bestehender Dienstleistungen zu erhöhen.

\subsection{Cyber-physische Systeme}

Im Rahmen dieses Beitrags werden digitalisierte, mit Sensorik und Konnektivität ausgestattete industrielle Maschinen und Anlagen, als CPS verstanden. CPS sind 
definiert als Systeme mit digitalen Komponenten wie Sensoren und Aktuatoren, die die physische Welt mit der digitalen Welt verschmelzen lassen (Lee 2008; Reinheimer und Strahringer 2014). Im Bereich der Informatik wurde der CPS-Begriff ursprünglich verwendet, um die Kopplung von Rechenleistung mit mechanischen Elementen zu beschreiben (Lee 2008). In seiner grundlegenden Studie identifiziert Lee (2008) die Voraussetzungen von CPS und beschreibt sie als Grundpfeiler der IT-Revolution im 20. Jahrhundert. Durch die von der deutschen Regierung ins Leben gerufene Initiative Industrie 4.0 gewinnt der CPS-Begriff auch in der IS Literatur zunehmend an Bedeutung (Böhmann et al. 2014; Matzner und Scholta 2014; Soeldner et al. 2013). Acatech, die deutsche Akademie der Technikwissenschaften definiert CPS als Systeme mit ,integrierter Software [...], die:

- mittels Sensoren physische Daten erheben und durch Aktuatoren direkt physische Prozesse beeinflussen können;

- aufgenommene Daten evaluieren, speichern und dadurch aktiv oder reaktiv mit der physischen oder digitalen Welt interagieren können;

- mit anderen Systemen in globalen Netzwerken via digitalen Kommunikationsfähigkeiten (kabelgebunden und/oder drahtlos, lokal und/oder global) vernetzt sind;

- global vorhandene Daten und Dienstleistungen nutzen können;

- eine Reihe von zugehörigen, multimodalen Schnittstellen von Mensch und Maschine besitzen“ (acatech - Deutsche Akademie der Technikwissenschaften 2011, S. 15).

Dieser Beitrag baut auf der oben dargelegten Konzeptualisierung von CPS und der Idee auf, digitalisierte Objekte und damit beispielsweise auch industrielle Maschinen und Anlagen als CPS zu sehen (Mikusz 2014; Schäfer et al. 2015). Durch die Nutzenpotentiale von CPS eröffnen sich neue Möglichkeiten für das wichtiger werdende industrielle Dienstleistungsgeschäft.

\section{Methodik}

Dieser Beitrag bietet einen strukturierten Überblick über die sich eröffnenden Nutzenpotentiale cyber-physischer Eigenschafen industrieller Maschinen und Anlagen für industrielle Dienstleistungen. Um möglichst allgemeingültige Erkenntnisse zu erzielen, wurden Fallstudien durchgeführt. Fokusgruppen-Workshops, Interviews und die Analyse interner Dokumente von Maschinen- und Anlagenbauern, Serviceorganisationen und Betreibern industrieller Maschinen und Anlagen bieten dabei eine breite Datenbasis. Notwendige Bedingung zur Auswahl der Organisationen war deren vorhandene Absicht, das Dienstleistungsgeschäft durch den Einsatz von cyber-physischen Elementen in Maschinen und Anlagen zu optimieren bzw. neue Dienstleistungen anzubieten. Zunächst wurden explorative Fokusgruppen-Workshops durchgeführt. Unter Einbeziehung der technischen und fachlichen Rollen und einer hohen Diversifizierung des Teilnehmerkreises wurde die Berücksichtigung verschiedener Perspektiven auf das Thema sichergestellt. In einem zweiten Schritt 
Tab. 2 Beispielhafter Szenariensteckbrief für einen konkreten Anwendungsfall

\begin{tabular}{ll}
\hline ID & 43 \\
\hline Titel & Wartungsvorhersage durch Nutzung des Rotationsgeräuschsensors \\
schreibung & $\begin{array}{l}\text { Block und Geitner (1983) stellten fest, dass 99\% aller Maschinenausfälle durch ge- } \\
\text { wisse Indizien oder den Zustand der Maschine vorangekündigt werden. In vielen Fällen } \\
\text { könnten die ersten Anzeichen einer Störung schon Wochen vor dem effektiven Ausfall } \\
\text { festgestellt werden. Die Veränderung der Maschinenperformance im Verlauf der Zeit } \\
\text { wird durch die P-f-Kurve aufgezeigt. Eine effektive Möglichkeit, um frühzeitig Abwei- } \\
\text { chungen vom normalen Betriebsmodus zu erkennen, ist beispielsweise bei rotierenden } \\
\text { Maschinen die kontinuierliche Überwachung mittels Ultraschall. Störungen können so } \\
\text { schon lange bevor sie der Mensch durch Berührung (Thermografie und Schwingung) } \\
\text { oder trainierte Ohren wahrnehmen kann, festgestellt werden. Beispielsweise können so } \\
\text { mögliche Ausfälle von Windturbinen in Windparks vor ihrem Auftreten mit Hilfe von } \\
\text { Rotations-und Vibrationssensoren sehr zuverlässig vorhergesagt werden. Die Ultraschall- } \\
\text { sensoren sind Teile cyber-physischer Systeme und können Abweichungen oder Ausfälle } \\
\text { der Windturbinen mit Hilfe der P-f-Kurve identifizieren }\end{array}$ \\
\end{tabular}

wurden zunächst halb-strukturierte Experteninterviews mit offenem Ende mit einer Dauer zwischen 50 und 110 min durchgeführt. Hierbei waren die Interview-Partner Service-Manager, Serviceprozessverantwortliche sowie Mitarbeiter der teilnehmenden Organisationen aus dem Innovationsbereich. Parallel wurden interne Dokumente, Präsentationen und Prozessdokumentationen durchleuchtet, um zusätzliche Anwendungsfälle zu identifizieren. Anschließend wurden standardisierte Szenariensteckbriefe genutzt, um konkrete Anwendungsfälle (Use Cases) mit den Experten zu diskutieren. Alle Interviews wurden aufgenommen. Auf Basis der Audio-Aufnahmen wurden Expertenkommentare in einer Datenbank abgelegt. Tabelle 2 zeigt beispielhaft einen konkreten Anwendungsfall.

Insgesamt wurden 45 Anwendungsfälle mit CPS im Kontext von Industriedienstleistungen identifiziert und mit den Experten diskutiert.

\section{CPS Nutzenpotentiale im Kontext industrieller Dienstleistungen}

Basierend auf den durchgeführten Fallstudien mit Industrieunternehmen und den 45 konkreten Anwendungsszenarien zur Nutzung cyber-physischer Fähigkeiten für industrielle Dienstleistungen wurden sieben Nutzenpotentiale abgeleitet. Tabelle 3 bietet einen Überblick über die identifizierten Nutzenpotentiale.

Im Folgenden werden die identifizierten Nutzenpotentiale im Detail vorgestellt. Für jedes Nutzenpotential wird exemplarisch ein konkretes Fallbeispiel dargestellt.

\subsection{Produktoptimierung durch die Auswertung von Betriebsleistungsdaten}

Operative Daten, die während des Betriebs industrieller Maschinen und Anlagen anfallen, können wertvolle Einblicke über die Nutzung und den Betrieb der Maschinen und Anlagen liefern. Durch einen Abgleich mit Reparatur- und Wartungsaufträgen können Schwachstellen der Maschinen und Anlagen im Feldbetrieb mühelos identifiziert werden. Häufig auftretende Probleme und Schwachstellen in der Konstruktion können auf Basis statistischer Analyse ex post identifiziert werden - entsprechende Gegenmaßnahmen können getroffen werden. 
Tab. 3 CPS Nutzenpotentiale im Kontext industrieller Dienstleistungen

\begin{tabular}{l} 
Nutzenpotential \\
\hline Produktoptimierung \\
durch die Auswertung von \\
Betriebsleistungsdaten \\
Betriebsoptimierung indust- \\
rieller Maschinen und Anlagen
\end{tabular}

Management und Steuerung industrieller Maschinen und Anlagen Kurzbeschreibung

Sensordaten von aktuell im Feld installierten Produkten können zur Entwicklung von optimierten Maschinen und Anlagen verwendet werden

Optimierung der Funktionsfähigkeit industrieller Maschinen und Anlagen durch die Auswertung von Sensordaten und entsprechender Optimierung. Ausfälle können verhindert werden. Durch das Generieren von Transparenz kann der Betrieb optimiert werden

Die Fähigkeit von CPS, Kontrollinformationen zu erhalten, ermöglicht es, industrielle Maschinen und Anlagen manuell über Servicecenter zu managen und zu steuern. Beispielsweise kann aus der Ferne ein Neustart der digitalen Komponenten der Anlage durchgeführt werden, um Fehler zu eliminieren

Zeitliche Vorhersage und Optimierung von TKD-Aktivitäten

Die kontinuierliche Datensammlung basierend auf CPS könnte für die Vorhersage und das Auslösen von Serviceaktivitäten des TKD genutzt werden. Zum Beispiel könnten routinemäßige Wartungsarbeiten auf der Grundlage von Messungen mittels integrierten Sensoren in den Maschinen und Anlagen durchgeführt werden

Ferndiagnose und Ersetzen von Aktivitäten des TKD

Oftmals können gewisse Wartungsarbeiten und sogar Reparaturen aus der Ferne gehandhabt werden. Servicezentren können aufgebaut und erfahrene Mitarbeiter diagnostizieren und lösen die Probleme nicht mehr vor Ort. Erfahrene Mitarbeiter können so effizienter eingesetzt werden, da sie nun vom Servicecenter aus tätig sind und nicht mehr vor Ort sein müssen. Ferndiagnosen werden gestellt und der TKD wird nur ausgesandt, falls das Problem nicht aus der Ferne gelöst werden kann

Optimierung und Unterstützung des Industrielle CPS können zur Optimierung und Unterstützung bestehender Dienstleistungen und Prozesse im TKD genutzt werden. Mit CPS können die TKD-Aktivitäten schneller erledigt werden und die Dienstleistungsqualität und -produktivität können gesteigert werden. Mitarbeiter des TKD beim Kunden vor Ort erhalten kontextabhängig detaillierte Informationen über den Zustand der Maschinen und Anlagen, sowie Unterstützung und Problemdiagnosen von erfahrenen Mitarbeitern aus dem Back-Office, um dadurch die Probleme vor Ort schneller und effizienter beheben zu können

Informations- und Datengetrie- Daten und Informationen von CPS können genutzt werden, um innobene Dienstleistungen vative datengetriebene Dienstleistungen anzubieten. Es ist beispielsweise denkbar, dass Maschinen- und Anlagenbauer Daten über den Betrieb bzw. Zustand der Maschinen und Anlagen an dritte Serviceorganisationen oder die Betreiber zu verkaufen

Beispielweise kann durch den Einsatz optimierter Materialien und Konstruktionen in der Aufzugsindustrie der Energieverbrauch während des Betriebs deutlich gesenkt werden. Die optimierten Produktvarianten sind weniger anfällig für Fehler und Ausfälle. Betriebsdaten müssen nicht mehr aufwändig durch Simulationen erhoben werden. Vielmehr können die realen, unverfälschten Daten von produktiven Maschinen und Anlagen im Feld genutzt werden. Im Rahmen einer gesamtheitlichen Betrachtung über den Produktlebenszyklus hinweg kann so die notwendige Wartungsintensität von Maschinen und Anlagen nachhaltig gesenkt werden. 


\subsection{Betriebsoptimierung industrieller Maschinen und Anlagen}

Aus der Perspektive des Produktlebenszyklus (Blinn et al. 2008) liegt das größte Potential cyber-physischer Fähigkeiten in der Betriebsphase von industriellen Maschinen und Anlagen. Gleichzeitig ergeben sich aus dem Betriebskontext aber auch die höchsten technischen Anforderungen, da hier der zeitliche Aspekt bei der Verarbeitung der Daten relevant ist. Gesammelte Daten und die Erkenntnisse können nicht nur im Kontext des TKD, sondern auch für die Verbesserung der gesamtheitlichen industriellen Betriebsabläufe innerhalb der Wertschöpfungsprozesses beim Anlagenbetreiber genutzt werden, in die die Maschinen und Anlagen eingebettet sind. Ein besseres Verständnis darüber, wie industrielle Maschinen und Anlagen im Feld von den Betreibern genutzt werden, kann wertvolle Hinweise auf mögliches Verbesserungspotential der Produkte liefern. Jedoch müssen in vielen Fällen historische Betriebsleistungsdaten zur Verfügung stehen, um statistische Analysen durchzuführen und das Potential für Verbesserungen aufzuzeigen.

Neben physischem Verbesserungspotential ist eine Optimierung der Maschinen und Anlagen beispielsweise auch durch Softwareupdates denkbar. Auf Basis historischer Daten können Hersteller durch Updates neue Funktionalitäten hinzufügen oder bestehende Funktionalitäten optimieren. Ist die Durchführung solcher Updates nicht möglich, kann beispielsweise durch die Herausgabe von Handlungsempfehlungen für Betreiber und Anwender eine Betriebsoptimierung herbeigeführt werden ${ }^{2}$.

\subsection{Management und Steuerung industrieller Maschinen und Anlagen}

Mit der Transformation von industriellen Maschinen und Anlagen in CPS wird das Equipment nicht nur mit Sensoren und einseitiger Konnektivität ausgestattet. Vielmehr wird eine bidirektionale Kommunikation ermöglicht. Aktuatoren in den Maschinen und Anlagen sind in der Lage, Steuerungsinformationen zu erhalten, sodass das Equipment aus der Ferne gesteuert und gemanagt werden kann. Serviceorganisationen sowie Maschinen- und Anlagenbauer können dadurch Betreibern der Maschinen produktbegleitende Mehrwertdienste anbieten, ohne physisch vor Ort zu sein. In der Aufzugsindustrie ist beispielsweise eine dedizierte Steuerung der Anlagen auf Basis externer Einflüsse (event-basierte Zugangskontrolle, Aufzugssteuerung in Abhängigkeit des Flugplans an Flughäfen) denkbar. Diese Beispiele stellen für den Betreiber der Anlage einen Zusatznutzen dar, sodass vom Hersteller und den Serviceorganisationen vergleichbare Funktionalität als Mehrwertdienstleistung angeboten werden können. Regelkreise zum Management und zur Steuerung von industriellen Maschinen und Anlagen erhalten dadurch eine deutlich höhere Auflösung. In diesem Zusammenhang wird auch von „High-Resolution Management“" gesprochen (Fleisch et al. 2014).

\footnotetext{
${ }^{2}$ General Electric zeichnet kontinuierlich Nutzungsdaten von Flugzeugturbinen auf. Da die effiziente Nutzung direkt von der Art und Weise wie Piloten das Flugzeug steuern beeinflusst wird, gibt General Electric nach Analyse der Nutzungsdaten konkrete Handlungsempfehlungen, wie sich der Betrieb effizienter gestalten lässt (http://www.gereports.com/post/91469336265/aerial-intelligence-this-airbus-makes-pilots, http://www.ge.com/europe/downloads/MM_CaseStudies_Aviation_Alitalia.pdf).
} 
Werden die neuen technologischen Möglichkeiten beispielsweise zum Management und zur Steuerung industrieller Maschinen und Anlagen aus der Ferne genutzt (Zolnowski et al. 2011), sind zur Realisierung vieler Anwendungsszenarien Echtzeitfähigkeiten zur Verarbeitung der Daten notwendig. Ebenfalls wäre die manuelle Fernsteuerung von industriellen Maschinen und Anlagen im Problemfall denkbar. Das Nutzenpotential zum Management und zur Steuerung des Equipments kann zur Effizienzsteuerung bestehender Dienstleistungsprozesse genutzt werden. Daneben ist aber auch die Entwicklung komplett neuer Dienstleistungsinnovationen denkbar, was zu zusätzlichen Umsatzpotentialen führen würde.

\subsection{Zeitliche Vorhersage und Optimierung von TKD-Aktivitäten}

Trotz der entstehenden Möglichkeiten zur Optimierung des Betriebs aus der Ferne und der Reduktion der Wartungsintensität sind für die Sicherstellung des Betriebs industrieller Maschinen und Anlagen Vor-Ort-Besuche durch den TKD unabdingbar. Grundsätzlich kann man drei verschiedene Wartungsstrategien unterscheiden: Korrektive Wartung (1), Präventive Wartung (2) sowie Preämptive Wartung (3) (Wang et al. 2007). Korrektive Wartung muss nach einem Ausfall vorgenommen werden, während Präventive und Präemptive Wartung vor dem tatsächlichen Ausfall durchgeführt werden. Im Falle der Präventiven Wartung kann man je nach Auslöser der Wartung zwischen drei verschiedenen Strategien unterscheiden. Im ersten Fall, der Zeitgesteuerten Präventiven Wartung (2a) wird die Wartungsaktivität von einem gesetzten Datum oder einem definierten Zeitpunkt ausgelöst. Der definierte Zeitpunkt der Wartung wird anhand von historischen Statistikdaten festgelegt (Alardhi und Labib 2007). In diesem Fall werden die Wartungsintervalle selten verbessert und Wartungsaktivitäten werden nicht effizient ausgelöst. Nutzungsbasierte Präventive Wartung (2b) beschreibt den Fall, dass Wartungsaktivitäten basierend auf der Intensität der Nutzung der Maschinen und Anlagen ausgelöst werden. Hierzu werden in der Regel keine automatisiert erhobenen operativen Daten der Maschinen oder Anlagen verwendet. Im dritten Fall, der Zustandsorientierten Wartung (2c), kann der Zustand der Maschinen und Anlagen Wartungsaktivitäten auslösen. Cyber-physische Komponenten ermöglichen die kontinuierliche und automatisierte Überwachung von Symptomen für das Versagen industrieller Maschinen und Anlagen. Bereits im Fall der zustandsorientierten Wartung tragen mit Sensorik ausgestattete, vernetzte Maschinen und Anlagen zu einer zeitlichen und qualitativen Optimierung der Wartungsaktivitäten bei. Der Zustand der Maschinen und Anlagen wird durch den Verschleiß der Teile definiert, wobei auf Basis der gesammelten Daten kein Forecasting betrieben wird. Neben den beschriebenen Möglichkeiten der Präventiven Wartung gibt es die Möglichkeit der Preämptiven Wartung (3). Bei dieser Methode werden die Daten ebenfalls aus dem operativen Betrieb der Maschine oder Anlage genutzt. Durch umfassende statistische Analysen der Sensordaten können zeitliche Trends hinsichtlich des Verschleißes einzelner Komponenten identifiziert und mögliche Ausfälle prognostiziert werden. Bloch und Geitner (1983) haben in diesem Zusammenhang aufgezeigt, dass das Versagen von industriellen Maschinen und Anlagen in den meisten Fällen auf Basis vordefinierter Kriterien von Symptomen vorhergesagt werden kann. Dieser Zusammenhang kann mit Hilfe der P-f-Kurve visualisiert wer- 
den (Wang et al. 2007). Kontinuierliche Datensammlung, basierend auf CPS, könnte somit als Vorhersage und Auslöser für Serviceaktivitäten genutzt werden. Effizienzsteigerungen durch digitalisierte und vernetzte industrielle Maschinen und Anlagen sind somit nicht nur während der Ausführung des Services möglich (vgl. Abschn. 4.6 Optimierung und Unterstützung des TKD), sondern auch bei der zeitlichen Planung der Wartungsintervalle. Im Kontext schwer erreichbarer Anlagen wie beispielsweise Offshore-Windparks können dadurch Anfahrtskosten eingespart werden und die Dienstleistungsproduktivität gesteigert werden.

\subsection{Ferndiagnose und Ersetzen von Aktivitäten des TKD}

Ein weiteres Nutzenpotential von digitalisierten industriellen Maschinen und Anlagen, die mit cyber-physischen Aktivitäten ausgestattet sind, liegt in den technischen Möglichkeiten, die es erlauben, Wartungsaktivitäten aus der Ferne durchzuführen. Mitarbeiter des TKD müssen so für bestimmte Aktivitäten nicht physisch vor Ort sein. Die Unternehmen der Fallstudien denken daher darüber nach, global agierende Servicezentren aufzubauen, sodass beispielsweise erfahrene Mitarbeiter Probleme aus der Ferne diagnostizieren und lösen können. Anfahrtswege und Stundensätze der TKD-Mitarbeiter im Feld sind teuer. Industrielle Maschinen und Anlagen werden zunehmend komplexer (Thomas et al. 2014). Im Vorfeld durchgeführte präzise Diagnosen, ohne vor Ort zu sein, erlauben eine Erhöhung der Dienstleistungsqualität der TKD-Beschäftigten im Feld. Darüber hinaus tragen umfangreiche vorgeschaltete Problemdiagnosen dazu bei, dass gerade unerfahrene Mitarbeiter im Feld genauer angeleitet werden und beispielsweise durch mobile Arbeitsunterstützungssysteme befähigt werden, auch komplexe Wartungs- oder Reparaturaktivitäten durchführen zu können (Herterich et al. 2015). Die Ferndiagnose ist das offensichtlichste Anwendungsszenario in diesem Kontext. In vielen Fällen kann die Wartung oder sogar die Behebung von Schäden aus der Ferne vorgenommen werden.

In der Aufzugsbranche wird dieses Nutzenpotential eingesetzt, um typische Routineaktivitäten des TKD vor Ort wie beispielsweise eine regelmäßige Überprüfung des Sicherheitsschaltkreises, der Notfallbatterie für die Kommunikations- und Steuerungstechnik, sowie das ordnungsgemäße Öffnen und Schließen der Türen als manuelle oder vollständig automatisierte Prozesse aus der Ferne durchzuführen, ohne dass Mitarbeiter des TKD zum Aufzug beim Betreiber vor Ort im Einsatz sein müssen.

\subsection{Optimierung und Unterstützung des TKD}

Für den größten Teil der Reparatur- und Wartungsdienstleistungen von industriellen Maschinen und Anlagen ist die physische Präsenz des TKD vor Ort notwendig. Als Beispiel kann der Austausch von Betriebsstoffen oder das Auswechseln defekter Komponenten angeführt werden. Cyber-physische Fähigkeiten können genutzt werden, um diese grundlegenden Dienstleistungen beim Kunden vor Ort zu optimieren und weiterzuentwickeln. Das Bereitstellen von auf der Basis von Sensordaten gewonnenen Erkenntnissen für den TKD ermöglicht eine höhere Dienstleistungsproduktivität und eine gesteigerte Dienstleistungsqualität. Auf Basis dieser Daten können konkrete Aktivitäten für den TKD abgeleitet werden und Probleme zielgerichteter 
gelöst werden. So kann ein Abgleich des Fehlerbilds einer konkreten Maschine oder Anlage mit historischen Fehlerbildern des gleichen Maschinentyps, sowie den in diesen Fällen durchgeführten Serviceaktivitäten erfolgen. Der in der Vergangenheit erfolgreich durchgeführte Wartungsauftrag lässt sich auf den aktuellen Fall projizieren. Dadurch kann eine Prognose über die konkret durchzuführenden Serviceaktivitäten abgegeben werden. Mit passender mobiler Unterstützung kann sich der TKD schon auf dem Weg zum Kunden einen Überblick über die Situation verschaffen und den Service zielgerichtet mit den entsprechenden Ersatzteilen durchführen. TKD Mitarbeiter haben so schon, bevor sie vor Ort sind, Zugriff auf relevante Sensordaten und Fehlercodes, anstatt erst lokal durch den Anschluss eines Diagnose-Geräts den aktuellen Zustand der Maschine oder Anlage einzusehen. Analytische Fähigkeiten und datengetriebene fachliche Unterstützung aus dem Backoffice helfen den Servicetechnikern bei der Durchführung des Serviceauftrags.

Um solche Szenarien umzusetzen, müssen gewisse Bedingungen erfüllt sein. Erstens müssen die Serviceprozesse einen hohen Grad an Standardisierung und Reife aufweisen. Nur so kann eine standardisierte und effektive Unterstützung durch mobile Arbeitsunterstützungssysteme erfolgen. Zweitens müssen operative Maschinendaten mit zusätzlichen Kontextinformationen weiterer Informationssysteme im Unternehmen (bspw. CRM, HR, ERP, sowie Systeme für den Einkauf von Ersatzteilen und zur Steuerung, Einsatzplanung und mobilen Unterstützung des TKD) angereichert werden; entsprechende Schnittstellen und ein gemeinsames Datenmodell müssen bereitgestellt werden. Ziel sollte dabei ein gesamtheitliches Datenmodell mit allen Serviceinformationen sein. All diese Informationen zusammenzutragen, erfordert jedoch ein hohes Investment und bringt viele Herausforderungen mit sich.

\subsection{Informations- und datengetriebene Dienstleistungen}

Die oben dargestellten Nutzenpotentiale zeigen, dass cyber-physische Eigenschaften industrieller Maschinen und Anlagen eine Vielzahl an Möglichkeiten bieten, bestehende industrielle Dienstleistungen und Geschäftsmodelle im Kontext von Reparatur und Wartung zu unterstützen und die Dienstleistungsproduktivität und -effizienz zu steigern. Zusätzlich zur Optimierung dieser bereits bestehenden Dienstleistungen und Prozesse können die entstehenden cyber-physischen Eigenschaften industrieller Maschinen und Anlagen auch grundsätzlich neue Nutzenpotentiale und Dienstleistungs-Geschäftsmodelle hervorbringen. Durch die eingebaute Konnektivität und Sensorik kann der Zustand der Maschinen und Anlagen zu jedem Zeitpunkt eingesehen werden. Ergänzt um die Möglichkeiten, Maschinen und Anlagen auch aus der Ferne zu steuern, sehen sich Akteure im Ökosystem mit der Macht über die Daten in der Lage, neue, datengetriebene Dienstleistungen anzubieten.

Betreiber industrieller Maschinen und Anlagen haben beispielsweise ein großes Interesse an Transparenz hinsichtlich Betrieb und Wartung. Darüber hinaus wollen die Betreiber von den Möglichkeiten profitieren, cyber-physische Maschinen und Anlagen in bestehende Wertschöpfungsprozesse zu integrieren und kontextabhängig Steuerungssignale versenden. Hierzu ist es aus Sicht der Hersteller bzw. Betreiber der Maschinen und Anlagen denkbar, standardarisierte Schnittstellen wie beispielsweise APIs anzubieten. Dies würde Betreibern die Möglichkeit geben, die Maschi- 
nen und Anlagen eines Herstellers mit weiteren Maschinen zu vernetzen, um so die Wertschöpfung gesamtheitlich zu optimieren. Dabei sind verschiedene Geschäftsmodelle denkbar. Beispielsweise könnten dritte Serviceorganisationen, die nicht nur ihre selbst hergestellten Anlagen warten und reparieren, den Zugriff auf bereits vorausgewertete Sensordaten sowie den digitalen Zugriff auf die Maschine als datengetriebene Dienstleistung erwerben. Ebenfalls denkbar aus der Perspektive der Betreiber wäre eine Abrechnung nach Nutzungsgrad der Maschinen und Anlagen bzw. eine ergebnisorientierte Bezahlung, statt den oft hohen Kaufpreis der Investitionsgüter bezahlen zu müssen. Die beispielhaft beschriebenen Szenarien haben gemeinsam, dass der Mehrwert allein auf den sich aus der Digitalisierung ergebenden Möglichkeiten erfolgt. Unternehmen, die traditionell Maschinen und Anlagen hergestellt und verkauft haben, entwickeln sich dadurch zu Datenlieferanten für ein Ökosystem, in dem komplett neue, produktbezogene Dienstleistungen entstehen.

\section{Implikationen und Handlungsempfehlungen für die Unternehmenspraxis}

Vor allem im Kontext der Initiative Industrie 4.0 wird die Digitalisierung im Maschinen- und Anlagenbau oftmals mit geschickt vernetzten Fertigungsprozessen zu Beginn des Lebenszyklus von industriellen Gütern assoziiert. Mit dem Trend der Serviceorientierung gewinnt in der Industrie jedoch die Betriebsphase der Maschinen und Anlagen zunehmend an Bedeutung. Maschinen- und Anlagenbauer suchen daher nach Möglichkeiten, innovative Dienstleistungen anbieten, die die Betriebsphase adressieren. Da die umfassende Digitalisierung der industriellen Güter und Serviceprozesse mit nicht zu unterschätzenden Kosten verbunden ist, ist es für Manager zunächst essentiell, schnell sichtbare Erfolge zu generieren. Kurzfristig liegt daher der Fokus auf den Möglichkeiten, die Effizienz und Qualität bestehender Dienstleistungen wie beispielsweise dem klassischen TKD auf Basis der cyber-physischen Fähigkeiten zu steigern.

Mittel- und langfristig wird hier jedoch eine Transformation stattfinden, da die gewonnenen Nutzenpotentiale über die angesprochenen Möglichkeiten hinaus komplett neue Dienstleistungen auf unterschiedlichen Ebenen ermöglichen. Beispielsweise könnten Industrieunternehmen in Kooperation mit Softwareanbietern die Analyse industrieller Sensordaten als Dienstleistung anbieten, die von dritten Unternehmen für die eigene Wertschöpfung genutzt wird. Durch diese Modularisierung entstehen Service-Ökosysteme; für Unternehmen wird es verstärkt erforderlich, mit Partnern zusammenzuarbeiten, da für die Erbringung komplexer Dienstleistungen oft ergänzende Dienstleistungen notwendig sind, die beispielsweise auf Grund fehlender Kompetenzen extern eingekauft werden müssen. In die Serviceprozesse müssen somit zunehmend mehr Akteure miteinbezogen werden. Sensorhersteller und Softwarefirmen sind nur zwei Beispiele. Durch Dienstleistungsinnovation gewinnen folglich Service Öksosysteme, Service Plattformen und, Value co-creation ${ }^{3}$ an Bedeutung (Lusch und Nambisan 2015).

\footnotetext{
${ }^{3}$ Value Co-Creation beschreibt die gemeinschaftliche Wertschöpfung durch den Einsatz von Ressourcen aller bei der Wertschöpfung beteiligter Akteure.
} 
Ferner ist auf Ebene der Geschäftsmodelle ein Paradigmenwechsel festzustellen: Unternehmen, die beispielsweise TKD-Leistungen anbieten, werden zunehmend nicht mehr nach tatsächlichem Aufwand für die Durchführung von Dienstleistungen bezahlt. Vielmehr rückt in Zukunft das Ergebnis in den Vordergrund - unabhängig von der aufgewendeten Zeit oder den Ressourcen zur Erbringung der Dienstleistung. Ebenfalls ist es denkbar, die Endprodukte als datengetriebene Dienstleistungen zu verkaufen oder dritte (Service-)Organisationen durch datengetriebene Dienstleistungen zu unterstützen. Beispielsweise denken Maschinen- und Anlagenbauer darüber nach, ihre Produkte als Dienstleistungen zu verkaufen - die Abrechnung könnte je nach Nutzungsintensität erfolgen. Durch neue Geschäftsmodelle und die Marktveränderungen könnte es in einigen Jahren unmöglich sein, industrielle Maschinen und Anlagen ohne die Nutzung der neuen Möglichkeiten herzustellen, zu betreiben oder zu warten. Trotz dieser Tatsache sollten Unternehmen sich nicht ausschließlich auf Dienstleistungen konzentrieren, die an die Betreiber gerichtet sind.

Bei Konzeption und Angebot innovativer Dienstleistungen sollten Unternehmen zunächst darauf achten, dass die Anwendungsfälle einfach umsetzbar sind, schnelle Fortschritte ersichtlich sind und diese direkt zu einer Erhöhung der Dienstleistungsproduktivität oder zur Generierung von zusätzlichem Umsatzpotential führen. Hoch standardisierte, repetitive Routine- und Kontrollaktivitäten, basierend auf der kontinuierlichen Zustandsüberwachung ohne fortgeschrittene analytische Fähigkeiten, sind beispielsweise einfach zu realisieren und sollten frühzeitig umgesetzt werden, um beispielsweise teure Servicetechniker im Feld einsparen zu können. Komplexe, datengetriebene mobile Unterstützung der Arbeitskräfte, für die eine Umgestaltung kompletter Prozessketten notwendig ist, sind aufgrund der Abhängigkeiten zwischen technischen und nicht-technischen Komponenten schwieriger umzusetzen und sollten auf Basis der grundlegenden Fähigkeiten zu einem späteren Zeitpunkt implementiert werden.

Trotz der fragmentierten Umsetzung sollten Praktiker dennoch ein umfassendes Verständnis für die Nutzenpotentiale haben, die CPS im Kontext industrieller Dienstleistungen generieren. Dieser Weitblick ist vor allem notwendig, um schon heute die richtigen Entscheidungen in Bezug auf die Entwicklung generativer und flexibler digitaler Plattformen und IT-Architekturen sowie einer tragfähigen Digitalisierungsstrategie zu treffen.

\section{Operative Maschinen- und Anlagendaten als zentrale Ressource für datenbasierte industrielle Dienstleistungen}

Ziel dieses Beitrags ist es, Nutzenpotentiale von CPS für das industrielle Servicegeschäft zu identifizieren und zu klassifizieren. Dazu wurden insgesamt elf Fallstudien mit Unternehmen aus dem Maschinen- und Anlagenbau, TKD-Serviceorganisationen, sowie Betreibern von industriellem Equipment durchgeführt. Die Identifikation und systematische Klassifikation konkreter CPS Anwendungsfälle für das industrielle Dienstleistungsgeschäft stellt einen ersten Schritt dar, um die Chancen und neu entstehenden technologischen Fähigkeiten für das industrielle Dienstleistungsgeschäft zu konkretisieren. 
In den heutigen kompetitiven und dynamischen Märkten können die Daten und die Erkenntnisse, die durch CPS gemacht werden, genutzt werden, um datenbasierende Dienstleistungsmöglichkeiten umzusetzen. In solchen Szenarien können traditionelle Herstellungsfirmen zu Datenverteilern für Serviceorganisationen oder operative Unternehmen werden. Zum Beispiel können die Daten, die im Besitz eines Maschinen- und Anlagenbauers sind, via standardisierter Interfaces an andere Akteure im Ökosystem verkauft werden. Diese wiederum können diese Daten für das Dienstleistungsgeschäft nutzen. Neben dem Verkaufen von Rohdaten (oder den aggregierten Daten) könnte der Besitzer der operativen Daten diese auch für einen zusätzlichen Service zu den traditionellen Wartungs- und Reparaturdienstleistungen verwenden. Nebst dem Gebrauch des CPS zur internen Effizienzsteigerung werden auch komplett neue Dienstleitungsmöglichkeiten geschaffen.

Der vorliegende Beitrag ist durch die angewandte Fallstudienmethodik limitiert. Es kann nicht sichergestellt werden, dass die identifizierten Nutzenpotentiale eine abschließende Zusammenstellung an Nutzenpotentialen für den adressierten Kontext darstellen. Eine Validierung und Ergänzung der präsentierten Ergebnisse sollte im Rahmen weiterer empirischer Forschungsvorhaben durchgeführt werden. Dennoch bietet der Beitrag aus praktischer Sicht einen Überblick über die durch CPS entstehenden Nutzenpotentiale für das industrielle Dienstleistungsgeschäft. Darüber hinaus kann der Beitrag als Ordnungsrahmen für künftige, tiefergehende Arbeiten in diesem Themenfeld genutzt werden.

Gleichzeitig wirft die vorliegende Arbeit jedoch auch neue Fragen auf, die auf einer konkreteren Ebene als zuvor in zukünftigen Forschungsvorhaben bearbeitet werden können. Durch die identifizierten Nutzenpotentiale wird die Relevanz des Themas aufgezeigt. In einem weiteren Schritt wäre es denkbar, die Erfolgsfaktoren und notwendigen technischen und organisationalen Fähigkeiten für die Adoption cyber-physischer Systeme im industriellen Kontext zu betrachten. TKD-Organisationen sind daran interessiert, die Effizienzpotentiale von CPS im Rahmen eines quantitativen Feldexperiments mit Mitarbeitern des TKD zu identifizieren. Hierbei ist der aktuelle Stand der Forschung zu mobilen Arbeitsunterstützungssystemen relevant (Herterich et al. 2015). Zur Erbringung von datengetriebenen Dienstleistungen im Ökosystem der beteiligten Akteure bedarf es digitaler Plattformen. Zukünftige Beiträge sollten sich dem Design solcher Plattformen als Wegbereiter für die Erbringung digitaler Dienstleistungen innerhalb von Ökosystemen beschäftigen. Abschließend ist festzustellen, dass der Einsatz von CPS die industrielle Dienstleistungserbringung stark verändern wird. Unternehmen benötigen zum einen Werkzeuge, die den Grad der Digitalisierung feststellen und erhöhen können. Neben den neu entstehenden Anwendungsmöglichkeiten und der Herausforderung, die entstehenden Potentiale durch entsprechende Umsetzungsstrategien zu nutzen, besteht zudem verstärkt der Bedarf an adäquaten Methoden für die Entwicklung von produktbezogenen digitalen Dienstleistungen.

Danksagung Dieser Beitrag entstand in Zusammenarbeit mit Partnerunternehmen des Kompetenzzentrums ,Industrial Services and Enterprise System ' (CC ISES) am Institute of Information Management der Universität St.Gallen. 


\section{Literatur}

acatech - Deutsche Akademie der Technikwissenschaften (2011) Cyber-Physical Systems: Innovationsmotor für Mobilität, Gesundheit, Energie und Produktion. Springer, München

Alardhi M, Labib AW (2007) Preventive maintenance scheduling of multi-cogeneration plants using integer programming. J Oper Res Soc 59(4):503-509

Barrett M, Davidson E, Prabhu J, Vargo SL (2015) Service innovation in the digital age: key contributions and future directions. MIS Quart 39(1):135-154

Blinn N, Nüttgens M, Schlicker M, Thomas O, Walter P (2008) Lebenszyklusmodelle hybrider Wertschöpfung: Modellimplikationen und Fallstudie an einem Beispiel des Maschinen-und Anlagenbaus,. In Proceedings of the Multikonferenz Wirtschaftsinformatik. München, S. 711-722

Bloch HP, Geitner FK (1983) Machinery failure analysis and troubleshooting, 2. Aufl. Gulf Publishing Co, Houston

Böhmann T, Leimeister JM, Möslein K (2014) Service systems engineering: a field for future information systems research. Bus Inf Syst Eng 6(1):73-79

Ebeling J, Friedli T, Fleisch E, Gebauer H (2014) Strategies for developing the service business in manufacturing companies. In: Lay G (Hrsg) Servitization in industry. Springer International Publishing, St. Gallen, S. 229-245

Fellmann DM, Kammler F, Reinke P, Matijacic M, Schlicker M, Thomas PDO et al. (2014) Nachfragebestimmte Spezifikation und Konfiguration mobiler Anwendungssysteme zur Steigerung von Produktivität und Empowerment im Technischen Kundendienst. In: Nüttgens M, Thomas O, Fellmann M (Hrsg) Dienstleistungsproduktivität. Springer Fachmedien Wiesbaden, Wiesbaden, S. 32-47

Fichman RG, Dos Santos BL, Zheng ZE (2014) Digital innovation as a fundamental and powerful concept in the information systems curriculum. MIS Quart 38(2):329-353

Fleisch PDE, Weinberger DM, Wortmann APDF (2014) Geschäftsmodelle im Internet der Dinge. HMD 51(6):812-826

Gebauer H (2008) Identifying service strategies in product manufacturing companies by exploring environment-strategy configurations. Ind Market Manag 37(3):278-291

Herterich M, Peters C, Uebernickel F, Brenner W, Neff A (2015) Mobile work support for field service: a literature review and directions for future research. In Proceedings of the 12th International Conference on Wirtschaftsinformatik (WI), Osnabrück

Kiritsis D (2011) Closed-loop PLM for intelligent products in the era of the internet of things. Comput Aided Des 43(5):479-501

Lee EA (2008) Cyber physical systems: design challenges. In Proceedings of the 11th International Symposium on Object Oriented Real-Time Distributed Computing (ISORC), Orlando, S. 363-369

Leimeister JM (2012) Dienstleistungsengineering und -management. Springer, Berlin

Lightfoot H, Baines T, Smart P (2013) The servitization of manufacturing: a systematic literature review of interdependent trends. Int J Oper Prod Man 33(11/12):1408-1434

Lusch RF, Nambisan S (2015) Service innovation: a service-dominant (SD) logic perspective. MIS Quart 1(39):155-175

Maglio PP, Spohrer J (2008) Fundamentals of service science. JAMS 36(1):18-20

Matzner M, Scholta H (2014) Process mining approaches to detect organizational properties in cyberphysical systems. In Proceedings of the 22nd European Conference on Information Systems (ECIS), Tel Aviv

Mikusz M (2014) Towards an understanding of cyber-physical systems as industrial software-productservice systems. PROCIR 16:385-389

Oliva R, Kallenberg R (2003) Managing the transition from products to services. Int J Serv Ind Manag 14(2):160-172

Reinheimer S, Strahringer S (2014) Cyber-physical systems - Von jeder mit jedem zu alles mit allem. HMD 51(6):810-811

Schäfer T, Jud C, Mikusz M (2015) Plattform-Ökosysteme im Bereich der intelligent vernetzten Mobilität: Eine Geschäftsmodellanalyse. HMD 52:1-15

Schoch T, Strassner M (2003) Wie smarte Dinge Prozesse unterstützen. HMD 229:23-32

Soeldner C, Roth A, Danzinger F, Moeslein K (2013) Towards open innovation in embedded systems. In Proceedings of the 19th Americas Conference on Information Systems (AMCIS), Chicago 
Thomas PDO, Nüttgens PDM, Fellmann DM, Krumeich J, Hucke S, Breitschwerdt DR et al. (2014) Empower mobile technical customer services (EMOTEC) - Produktivitätssteigerung durch intelligente mobile Assistenzsysteme im Technischen Kundendienst. In: Nüttgens M, Thomas O, Fellmann M (Hrsg) Dienstleistungsproduktivität. Springer Fachmedien Wiesbaden, Wiesbaden, S. 2-17

Ulaga W, Reinartz WJ (2011) Hybrid offerings: how manufacturing firms combine goods and services successfully. J Marketing 75(6):5-23

Vargo SL, Lusch RF (2008) Service-dominant logic: continuing the evolution. JAMS 36(1):1-10

Wang L, Chu J, Wu J (2007) Selection of optimum maintenance strategies based on a fuzzy analytic hierarchy process. Int J Prod Econ 107(1):151-163

$\mathrm{Xu}$ R, Ilic A (2014) Product as a service: enabling physical products as service end-points. In Proceedings of the 35th International Conference on Information Systems (ICIS), Auckland

Yoo Y, Henfridsson O, Lyytinen K (2010) The new organizing logic of digital innovation: an agenda for information systems research. Inform Syst Res 21(4):724-735

Zolnowski A, Schmitt AK, Böhmann T (2011) Understanding the impact of remote service technology on service business models in manufacturing: from improving after-sales services to building service ecosystems. In Proceedings of the 19th European Conference on Information Systems (ECIS), Helsinki 